\title{
THE SURVEILLANCE OF COMMUNICABLE DISEASES IN THE EUROPEAN UNION - A LONG-TERM STRATEgy (2008-2013)
}

\author{
A Amato-Gauci (andrew.amato@ecdc.europa.eu)1, A Ammon ${ }^{1}$ \\ 1. Surveillance Unit, European Centre of Disease Prevention and Control, Stockholm, Sweden
}

\begin{abstract}
This article presents the steps and considerations that led to the development of the European Centre for Disease Prevention and Control's (ECDC) long-term strategy for the surveillance of communicable diseases in the European Union (EU) for the years 2008 to 2013 [1]. Furthermore, it outlines the key features of the strategy that was approved by the ECDC's Management Board in December 2007
\end{abstract}

Why is it necessary to carry out surveillance at the European level?

National surveillance systems and methods are very diverse and the quality of data collated varies across the EU and the three participating countries of the European Economic Association/ European Free Trade Association (EEA/EFTA). This diversity is not limited to different data collection and validation systems and different reporting systems but even to basic issues such as different interpretations of the same standard case definitions. There are also country- specific variations in the organisation of health-care systems and in the availability of facilities and equipment for diagnostics and case confirmation, all of which also contribute to this diversity. As a result, the data produced are often not comparable, as was recently demonstrated in the ECDC's first Annual Epidemiological Report on Communicable Diseases in Europe [2,3].

This diversity also applies to the 17 EU-wide Dedicated Surveillance Networks (DSNs) [4], some of which were established as early as the 1980s. They differ in scope and coverage, objectives, structure of organisation, and development phase. They have developed separate reporting rules and procedures, variable data validity checks and all have their own separate report layouts. Therefore, a more coordinated approach towards surveillance at the European regional level should lead to a better harmonisation of structures and improve the comparability of the data and hence provide an added value for all EU Member States (MS) and EEA/ EFTA countries (Table 1).

\section{Types of surveillance}

Several definitions of surveillance of health and disease have been published by a number of authors $[5,6,7]$, with only slight variation between them. All these definitions incorporate the main elements of ongoing data collection, analysis to convert this data into statistics, interpretation of this analysis to produce information and then dissemination of this information to those who can take appropriate action.
In the context of the ECDC's work, surveillance is defined as the ongoing collection, validation, analysis and interpretation of that health and disease data that is needed to inform key stakeholders (in MS and elsewhere) to permit them to take action by planning and implementing more effective, evidence-based public health policies and strategies relevant to the prevention and control of disease or disease outbreaks. The prompt dissemination of the information to those who need to know is as essential as ensuring the quality, validity and comparability of the data.

\section{Indicator-based surveillance}

The traditional approach to the surveillance of communicable disease consists of routinely collecting data about the occurrence of predefined diseases, specific pathogens, syndromes or conditions from health-care providers. This notification process relies on standard case definitions for surveillance to ensure a uniform approach to reporting by all clinicians and laboratories and to improve the comparability of the data and reports across healthcare services. The notifications are then routinely compiled and analysed to produce indicators that could suggest the existence of a threat or a problem that needs addressing. In some cases, a public health intervention would be required from the notification of a

\section{T A B L E}

The main European Union added value of a more coordinated approach to surveillance
1. Improve inter-country comparability of data through a number of initiatives including by promoting the correct application of standard case definitions;
2. Reduce complexity in surveillance systems across Europe;
3. Avoid duplication of work through double reporting with various European organisations;
4. Provide more relevant and reliable data to produce higher quality public health evidence;
5. Strengthen the national surveillance systems by contributing to capacity building and standards setting in the countries;
6. Enhance the detection and monitoring of international outbreaks;
7. Be economically more efficient and sustainable in the long term than the disease-specific projects based system;
8. Allow easier access to and use of the data by all who may need it;
9. Better facilitate the inclusion of diseases into the surveillance and general research agenda according to the European priorities. 
single case of the disease while in other situations, a threshold may be applied to an indicator to show up an unusual incidence rate of the disease in a given community. This "indicator-based" approach has proved to be very effective in monitoring threats related to known risks and then in ensuring the prompt implementation of public health measures.

While this traditional approach remains the backbone of public health surveillance for communicable diseases, it has proven to be less effective in ensuring prompt recognition of emerging problems. Several further approaches seek to complement traditional surveillance in order to enhance its ability to detect pubic health threats. Some of these, such as syndromic surveillance or activity monitoring, remain heavily reliant on the routine collection of structured data, again compiled as indicators. Inclusion of these approaches would only be done after discussion and agreement with MS.

\section{Event-based surveillance}

A novel approach takes advantage of the availability of advanced information technology by scanning such sources as the Internet and media continuously to detect information that may lead to the recognition of emerging threats. This "event-based" surveillance [8] approach has been introduced to complement effectively the indicator-based surveillance approach. It uses unstructured data, that then needs to be studied and verified and cannot be summarised as an indicator.

Together, both these approaches can conveniently be referred to as gathering strategic information on disease.

Steps towards a coordinated approach to surveillance in the EU

In 2005, a strategy for infectious disease surveillance in Europe was finalised to outline the transition phase from the existing project-based approach of the DSNs, mainly led by the Commission, to a more coordinated and sustainable one coordinated by the ECDC. Following this, the ECDC planned to develop a longer-term vision of the future surveillance of communicable diseases in the $E U$, to better ensure a common understanding of the direction and the decisions needed for the further development of the European wide surveillance systems. The drafting of this document took into account the ECDC's emergent strategy on how it will be developing the future work with laboratories, to ensure synergy across the organisation.

\section{Goals of the ECDC's long-term surveillance strategy}

The strategy defines the terms and scope of surveillance, broad goals and objectives, the organisational requirements, support needs for the MS and outlines a roadmap to implement the strategy. The overall goal of these surveillance activities is to contribute to reducing the incidence and prevalence of communicable diseases in Europe by providing relevant and accurate public health data, information and reports to decision makers and health-care professionals in an effort to promote actions that will result in the timely prevention and control of communicable diseases in Europe. Good comparability of surveillance data between MS and a high validity of communicable disease data is a key component dictating the success of this goal.

In order to achieve these goals, both the ECDC and MS have to work in close partnership to build up a strong surveillance system on the European level. MS need to strengthen, maintain or set up the structures which are required to provide the relevant data - in certain cases this may require support from the ECDC. At the same time, the ECDC will continue to develop the infrastructure and common framework, including quality assurance systems and training support, required at the EU level.

There are a number of areas where further work will be essential. These include revising the case definitions for surveillance on the EU level [9] and having a mechanism for occasional review; introducing clear principles of collaboration on data exchange, access and publication acceptable to all MS and the ECDC; ensuring a regular review of disease-specific surveillance objectives and priorities following wide consultation; developing links to other existing international databases; developing systems to critically review the diseases under EU-wide indicator based surveillance; planning for the greater integration of data from laboratories and developing ways of improving collaboration with them, in particular with the national reference level laboratories (NRL); developing more advanced data analysis methods and studying how best to communicate the results to ensure that this is information used for action.

Apart from these activities, several initiatives and systems will be essential to the success of this strategy.

\section{The European Surveillance System}

The ECDC has developed an information system for infectious disease indicator-based surveillance at the European level, The European Surveillance System (TESSy). TESSy will be a valuable tool to improve the collection, validation, storage and dissemination of surveillance data of the EU MS and EEA/EFTA countries. MS are already using it with the collection of a reduced set of common variables important for the routine surveillance of cases of all infectious diseases. TESSy will enable:

- Standardising data collection on infectious diseases surveillance;

- Providing a 'one-stop shop' for reporting and retrieving data for the MS;

- Standardising the basic reports based on surveillance data;

- Providing a consistent and easily available overview of the current situation in the EU.

\section{Epidemic intelligence}

Another system being developed focuses on developing event-based surveillance to better provide epidemic intelligence information [10]. The ECDC is working to ensure that all countries have standard procedures and tools in place to monitor and assess threats detected early. Similarly all countries will be able to use the ECDC developed 'Threat Tracking Tool' to perform joint risk assessments in the event of a threat potentially affecting more than one country. Finally the epidemic intelligence system will enable MS to continue to routinely report communicable disease threats through the Early Warning and Response System (EWRS) [11] once their assessment has confirmed the existence of a threat affecting the EU (as defined in the EWRS regulations).

\section{Partnerships}

Various collaborative agreements will be finalised with the other regional organisations also involved in the surveillance of disease, such as the World Health Organization (WHO) Regional Office for Europe and their global office in Geneva, the European Food Safety Authority (EFSA) and the European Monitoring Centre for Drugs and Drug Addiction (EMCDDA), in order to minimise duplication 
and ensure that activities are complementary. Agreements on the principles of collaboration on data exchange between the ECDC and MS will be developed to define clearly the role of data providers and data users both in MS and the ECDC (and other parties, e.g. $\mathrm{WHO}$ ) and the procedures for publishing the results of the analysis of data.

\section{Collaboration with the Member States}

The future collaboration with disease-specific experts in MS nominated by the ECDC's Competent Bodies, will be structured by a division of the diseases/pathogens into six main groups, namely respiratory tract infections; sexually transmitted infections, including HIV and blood-borne viruses; food- and water-borne diseases and zoonoses; emerging and vector-borne diseases; vaccine-preventable diseases; and antimicrobial-resistant pathogens and healthcareassociated infections. Where necessary, more focussed (diseasespecific) subgroups can be established within any of these six groups. Annual meetings will be held for each of these six main groups to discuss issues pertinent to the surveillance of the whole disease group. If needed, specific 'parallel session' symposia can be held at the same time. For each of these six groups, a small Coordinating Group will be established and take over many of the functions carried out by the former DSN steering groups.

The ECDC plans to support the capacity development of MS to strengthen their surveillance by providing training, country visits to deal with MS-specific issues (including needs assessments and exploring ways to strengthen national systems), quality assurance (and EQA) and control processes, protocols, SOPs, guidelines, etc. Furthermore, the ECDC will work to strengthen the laboratory capacity in the EU and EEA/EFTA countries and the candidate countries in collaboration with the Commission, the ECDC Competent Bodies, and nominated National Microbiology Focal points, to ensure that every country should have the capacity of, or at least have the access to, Reference Level Laboratory (NRL) services enabling them to confirm the diagnosis, isolation of and further characterisation of all the important pathogens.

\section{Implementation}

The strategy will be implemented in two phases: a transition period until 2010, when the main focus will be on the integration of the coordination of the current DSNs to the ECDC while consolidating its own technical capacity; and the period between 2010 and 2013 when the ECDC will have taken over the full responsibility of surveillance and can then focus on developing and consolidating the highest quality and effective system possible for Europe. In order to keep this strategy and its objectives relevant, it will be revisited from time to time, with the Commission, MS and key stakeholders, so that it may be adjusted to incorporate emerging strategies or new evidence as required.
4. EU surveillance networks. Available from: http://ecdc.europa.eu/Links.html 5. Heymann DL (editor). Control of Communicable Diseases Manual, 18th edition. APHA, 2004.

6. Eylenbosch WJ, Noah ND, (editors). Surveillance in Health and Disease. Oxford University Press, 1988.

7. Last JM (editor). A Dictionary of Epidemiology, 4th edition. Oxford University Press, 2001.

8. Paquet C, Coulombier D, Kaiser R, Ciotti M. Epidemic intelligence: a new framework for strengthening disease surveillance in Europe. Euro Surveill. 2006;11(12):pii=665. Available from: http://www.eurosurveillance.org/ ViewArticle.aspx?ArticleId $=665$

9. European Commission Decision of 8/IV/2008. Available from: http://ecdc.europa. eu/Activities/surveillance/Pdf/Revised_case_definitions1589_2008_en.pdf

10. Coulombier D. Epidemic intelligence in the European Union: strengthening the ties. Euro Surveill. 2008;13(6):pii=8030. Available from: http://www. eurosurveillance.org/ViewArticle. aspx?ArticleId $=8030$

11. Guglielmetti P, Coulombier D, Thinus G, Van Loock F, Schreck S. The Early Warning and Response System for communicable diseases in the EU: an overview from 1999 to 2005. Euro Surveill. 2006;11(12):pii=666. Available from: http://www.eurosurveillance.org/ViewArticle.aspx?ArticleId=666

This article was published on 26 June 2008.

Citation style for this article: Amato-Gauci A, Ammon A. The surveillance of communicable diseases in the European Union - a long-term strategy (2008-2013). Euro Surveill. 2008;13(26):pii=18912. Available online: http://www.eurosurveillance.org/ ViewArticle.aspx?ArticleId $=18912$
References

1. Surveillance of communicable diseases in the European Union, a long-term strategy: 2008-2013. European Centre for Disease Prevention and Control. Available from: http://www.ecdc.europa.eu/documents/pdf/Surveillance_of CD_EU.pdf

2. Annual Epidemiological Report on Communicable Diseases in Europe. European Centre for Disease Prevention and Control. June 2007. Available from: http://www.ecdc.europa.eu/pdf/ECDC_epi_report_2007.pdf

3. Amato-Gauci A, Ammon A. ECDC to launch first report on communicable diseases epidemiology in the European Union. Euro Surveill. 2007;12(23):pii=3213. Available from: http://www.eurosurveillance.org/ViewArticle.aspx?ArticleId=3213 